\title{
SECONDARY USER UNDERCOVER COOPERATIVE DYNAMIC ACCESS PROTOCOL FOR OVERLAY COGNITIVE RADIO NETWORKS
}

\author{
A. Masri ${ }^{1}$, Y. A. S. Dama ${ }^{1,2}$, N Eya ${ }^{2}$, R. A. Abd-Alhameed ${ }^{2}$, N. Odeh ${ }^{1}$, F.Hasan $^{1}$, \\ A. Mousa ${ }^{1}$, and J. M. Noras ${ }^{2}$ \\ ${ }^{1}$ Dept. of Telecommunication Engineering, An-Najah National University, Nablus, \\ Palestine \\ ${ }^{2}$ School of Engineering and Informatics, University of Bradford, Bradford, UK
}

\begin{abstract}
A secondary cooperative overlay dynamic spectrum access protocol in cognitive radio networks is proposed, allowing secondary users to access the primary system using full power without causing harmful interference to primary users. Moreover, an enhancement in the primary system will be achieved as a result of secondary relaying of primary messages. A detailed description of the protocol is given and illustrated with network scenarios.
\end{abstract}

\section{Keywords}

Cognitive Radio, Dynamic Spectrum Access, Resource Management, Cooperative Overlay.

\section{INTRODUCTION}

Recently a new regulatory model has been considered to open licensed (Primary) frequency spectrum to unlicensed (Secondary) access, with the aims of improving spectrum utilization, and freeing up space for new radio technologies. This new model requires the development of Cognitive Radio (CR) devices that are able to access the primary bands without causing any harmful interference to Primary Users (PUs). Several such schemes have been suggested, which can be classified as Interweave, Underlay or Overlay access paradigms [1].

The Overlay access scheme authorizes secondary and primary concurrent access over the same channel; the secondary power is split into two parts, one for the secondary access link and the other part to relay or assist the primary communication $[2,3,4,5]$. With the correct choice of the power splitting ratio, the potential reduction in a PU's Signal to Interference plus Noise Ratio (SINR) due to the secondary user is exactly compensated by the relaying assistance power.

Although the overlay access paradigm can outperform other paradigms in terms of capacity, it suffers from several practical limitations [3, 6, 7], of which the most stringent is that the Secondary User (SU) has to maintain synchronization with the primary system to guarantee seamless secondary co-existence within it. Such a synchronization requirement is already achieved for the DVB-T Single Frequency Network (SFN) based Overlay CR in [4], where the primary signal is sent via satellite to major transmitters which apply the required delays. Thus, a potential secondary transmitter might also gain access to the primary signal, keeping time and frequency synchronization with the primary transmitters and therefore join the primary network using the Overlay scheme [8].

DOI : 10.5121/ijmnct.2017.7201 
Recent work on the Overlay $\mathrm{CR}$ access paradigm in $[1-4,6,7,9,10]$ assumes perfect synchronization between secondary and primary users, and adopts the simple network scenario shown in Fig. 1. In contrast, the recent work of Marsi et al. [5], proposed a distributed synchronization protocol that ensures that secondary users are synchronized with the primary transmissions: however, this is also applied in the ideal scenario of Fig. 1.

In the conventional overlay cognitive radio scheme, described above, SUs must allocate power to compensate the interference caused by their presence so it may arise that the remaining power is not adequate for reliable transmission of secondary data [2]. In such cases, the secondary is forced to keep silent and lose a transmission opportunity. More generally, the conventional overlay protocol offers no advantage to the primary system, which will make the existence of secondary system within the primary system unattractive to primary regulators.

Based on the idea of synchronization work in [5], in this paper we propose a dynamic spectrum access overlay protocol which we call the Secondary Undercover (SUC) protocol. We extend the network scenario to contain several SUs that work together cooperatively in order to access the primary band, not only without causing harmful interference to PUs, but also using relaying to improve the primary system performance. We target secondary access to primary spectrum, in which PUs utilize Hybrid Automatic Repeat Request (HARQ) and Orthogonal Frequency Division Multiplexing with Cyclic Prefix (OFDM-CP).

The rest of this paper is organized as follows. Description of the system model is presented in section two. In section three, we describe briefly the synchronization protocol, while in section four we elaborate the Secondary Undercover protocol. We present the performance of our proposal in section five, and finally, in section six draw some conclusions and propose future work.

\section{SYSTEM MODEL}

Fig. 1 describes the basic network setup which represents the relation between pairs of SUs and targeted PUs. We extend this setup to contain several SUs as in Fig. 2 and Fig. 4. All users are assumed to be within the transmission range of each other and they are ordered in line of sight with the primary users to simplify the model. The fading between the users is flat quasi-static

Rayleigh; using the per block-fading channel model, effective channel gains $\left|h_{i j}\right|^{4}$ are exponentially distributed. We assume that the active SU applies no rate control and uses a transmission power of maximum value equal to $P_{s}$. Moreover, a feedback channel exists between SUs.

We adopt the overlay based HARQ protocol in Fig. 3 for the setup in Fig. 2, to obtain prior knowledge about the PU's message [2], which is required to perform the overlay access. In contrast to conventional overlay access, instead of letting each secondary transceiver compensate for its interference by splitting its power and relaying the primary message, we propose a different method in section four that takes into account the compensation process. PUs are assumed to use OFDM-CP. 
Table 1

\begin{tabular}{|c|c|}
\hline Symbols & Description \\
\hline$T_{A C K}, T_{N A C K}$ & Time required for receiving an $\mathrm{ACK} N \mathrm{NACK}$ \\
\hline$T_{M s g}$ & Time required for receiving a PU's message \\
\hline$d_{i j}$ & The Euclidean distance between user ${ }^{i}$ and $j$ \\
\hline$T_{p_{i j}}$ & The propagation delay between user $\mathrm{i}$ and $\mathrm{j}$ \\
\hline $\boldsymbol{C}$ & Speed of light \\
\hline$\Delta T$ & $\begin{array}{l}\text { Time difference between the primary and secondary start } \\
\text { transmission }\end{array}$ \\
\hline$T_{w}$ & $\begin{array}{l}\text { Time between receiving a message and transmitting an } \\
\qquad \mathrm{ACK} N \mathrm{NCK} \text { back }\end{array}$ \\
\hline$T_{m}$ & $\begin{array}{l}\text { Time between receiving an } \mathrm{ACK} / \mathrm{NACK} \text { and } \\
\text { transmitting retransmitting a message }\end{array}$ \\
\hline$t_{k}^{0}$ & $\begin{array}{l}\text { Primary start transmitting/retransmitting message time and } \mathrm{k} \\
\text { is the message sequence number }\end{array}$ \\
\hline$t_{k}^{j i}$ & Start receiving time on user $i$ from user $j$ \\
\hline$\widehat{T}$ & $\begin{array}{l}\text { Secondary estimated waiting time a fter receiving a NACK } \\
\text { and before start transmission }\end{array}$ \\
\hline$\alpha$ & Secondary power ratio \\
\hline
\end{tabular}

As a consequence of multipath mechanisms, the variations of the received signal power strength will be characterized by path loss and shadowing effects. The signal is said to experience flat or frequency selective fading, dependent upon the estimated delay spread value. The proposed model was tested over different values of delay spread depending on the propagation environment.

We could adopt the overlay based SFN DTV as in Fig. 4, in which the SUs obtain prior knowledge about the primary data directly from the SFN DTV network. However, in this paper we work within the more challenging setup using HARQ as in Fig. 2. Moreover, in this work we do not address how the secondary receiver extracts its data from all received messages.

Next, we describe briefly the distributed synchronization protocol to be used as a basis for the proposed access protocol.

\section{Synchronization Between Primary AND Secondary TRANSCEIVERS}

Table 1 describes the terminology used in this paper. The system model was described in section 2 , and SUs are assumed to have access to a geolocation database which will provide location information about the PU transmitter (usually a base station), but no information about 


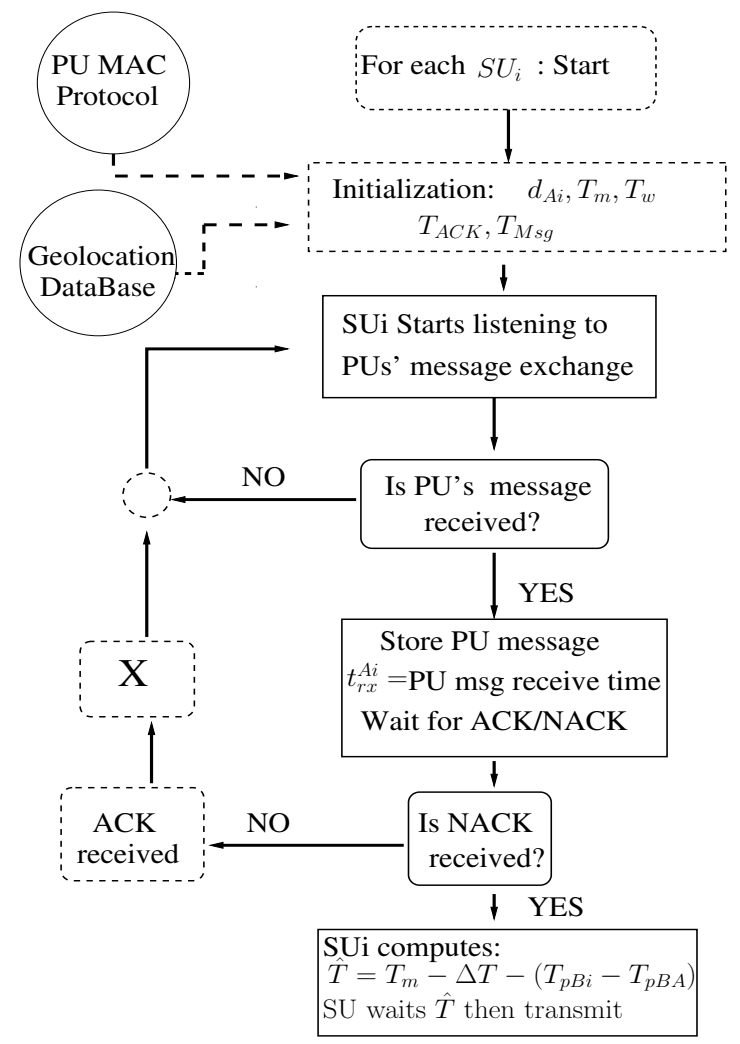

Fig. 5 Cooperative overlay protocol flow graph.

PU receiver [11]. In addition, $S U_{i}$ has full knowledge about the PU's HARQ MAC protocol information.

$S U_{i}$ initiates the synchronization protocol by following the flow graph in Fig. 5, thus obtaining the initialization parameters $\left\{a_{A i}, T_{m,}, T_{w}, T_{A C R}, T_{M s g}\right\}$ from the PU's MAC protocol and the geolocation database. Subsequently, $S U_{i}$ keeps listening for the primary channel to which it would like access, checking if a PU's message is transmitted. If so, it will store this message and record the start reception time ${ }^{t / i i}$, then wait for an ACK/NACK from the PU receiver.

SUi deletes PU's message
$t_{r x}^{B i}=$ PU ACK receive time
SUi computes:
$\Delta T=T_{m}-\left(t_{r x}^{A i}-T_{p A i}-t_{r x}^{B i}-T_{A C K}\right)$
$t_{2}^{0}=t_{r x}^{A i}-T_{p A i}$
$T_{p_{B A}}=T_{p_{A B}}=\left(t_{2}^{0}-T_{m}-T_{A C K}-T_{w}\right)-\left(t_{1}^{0}+T_{M s g}\right.$
$T_{p B i}=T_{p A B}+\Delta T$

Fig. 6 Computations required by SUi before starting transmission. 
If an ACK is received then $5 U_{i}$ knows that there will be no retransmission of the stored message, so $5 U_{i}$ deletes the stored message and performs the computations shown in Fig. 6. If a NACK is received, then $S U_{i}$ computes the time $\hat{T}$ seconds that it should wait before starting its transmission concurrently with the primary one, to guarantee synchronous reception on the PU receiver with the message from the PU transmitter. This is true as long as we maintain a relative delay between the secondary relaying symbol and primary symbol that does not exceed the duration of the cyclic prefix of the primary transmitter signal [12].
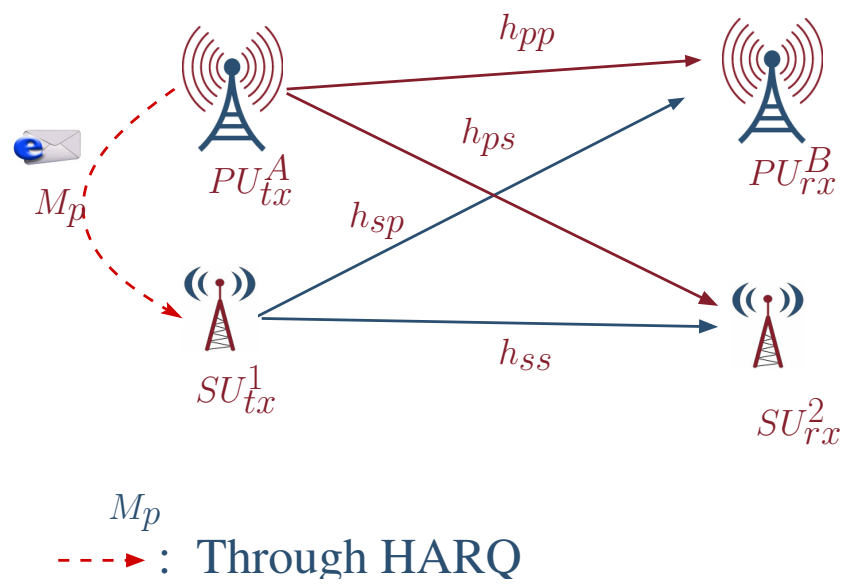

Fig. 1 Network scenario with two primary and secondary pairs is presented. The dotted line indicates a priori knowledge of the PU's message at the SU transmitter.

Using the simple network scenario in Fig. 1 and as one example scenario we assume that primary transmitter $P U_{t w i}^{A}$ is closer to $\mathrm{SU}$ transceiver $S \|_{i, \pi}^{i}$ than to the primary receiver $P U_{m \infty}^{E}$, (i.e; $d_{A i}>$ $\left.d_{A B}\right)$, then Fig. 7 shows the simulated example scenario and how the SU is able to compute the correct value of $\hat{T}$ to guarantee synchronous reception with the primary signal on the primary receiver. Next, we describe our cooperative dynamic access protocol.

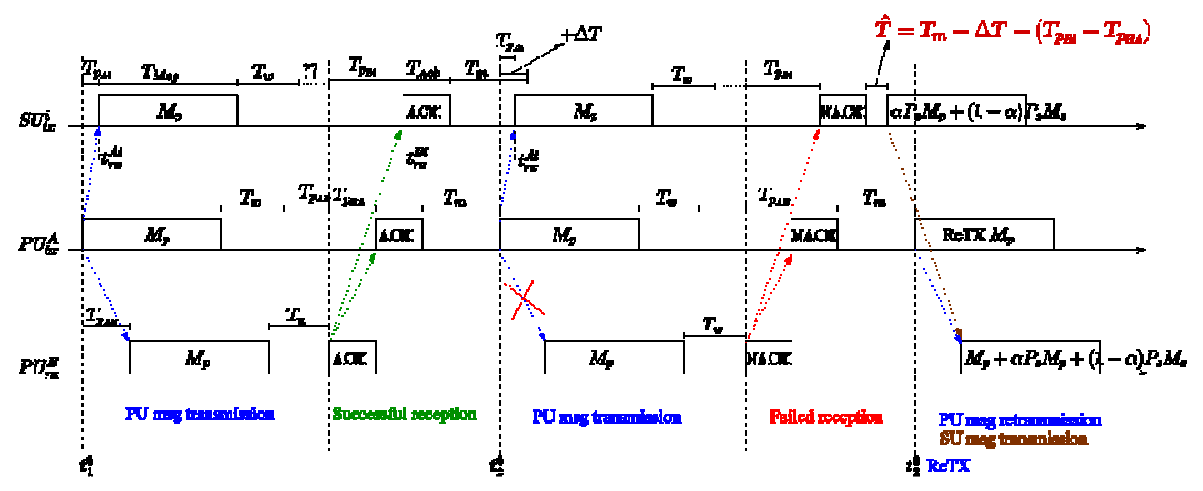

Fig. 7 Transmission scenario using HARQ as the source of primary data for secondary system, without using the SUC protocol and SU is responsible for interference compensation. 


\section{Secondary User Under Cover (Suc) Protocol}

As explained in the introduction, the conventional overlay cognitive radio access forces SUs to split their power and thereby lose transmission opportunities [2]. In contrast, the SUC protocol lets the SU transmit data using full power, while it delegates the interference compensation to other SUs who cooperate to cover the secondary data transmission by relaying the known primary message as shown in Fig. 2 and Fig. 4. In this way, any potential decrease in the PU's SINR due to secondary transmission is compensated. In fact, the PU's SINR could be improved by increasing the number of SUs in the system, which would make the existence of a secondary system within the primary system preferable to primary regulators.

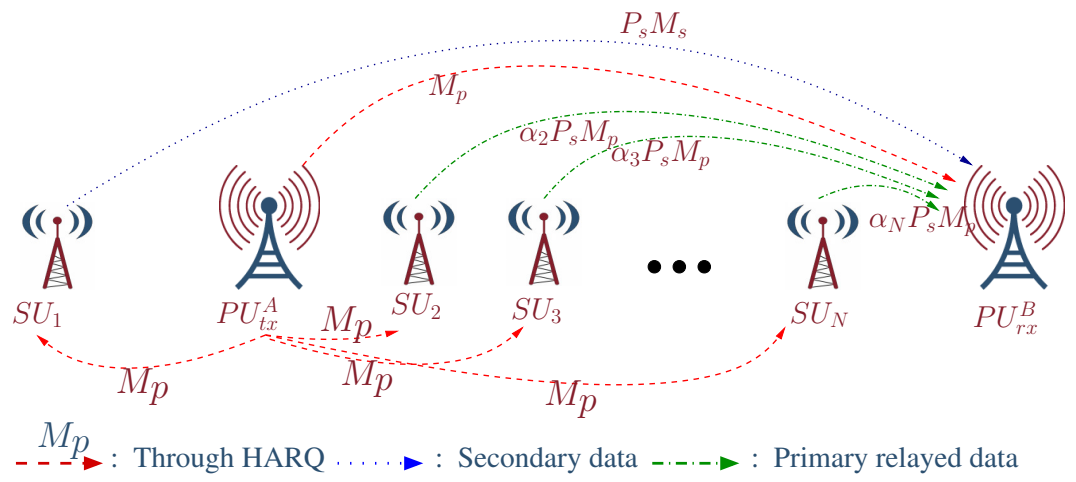

Fig. 2 Extended network scenario with two PUs and multiple SUs is presented, using HARQ as the source of primary data for secondary system.

In our protocol, we assume that SUs have a prior agreement about who will transmit secondary data and who will be in support at each transmission opportunity. Fig. 8 presents the flow graph of the SUC protocol.

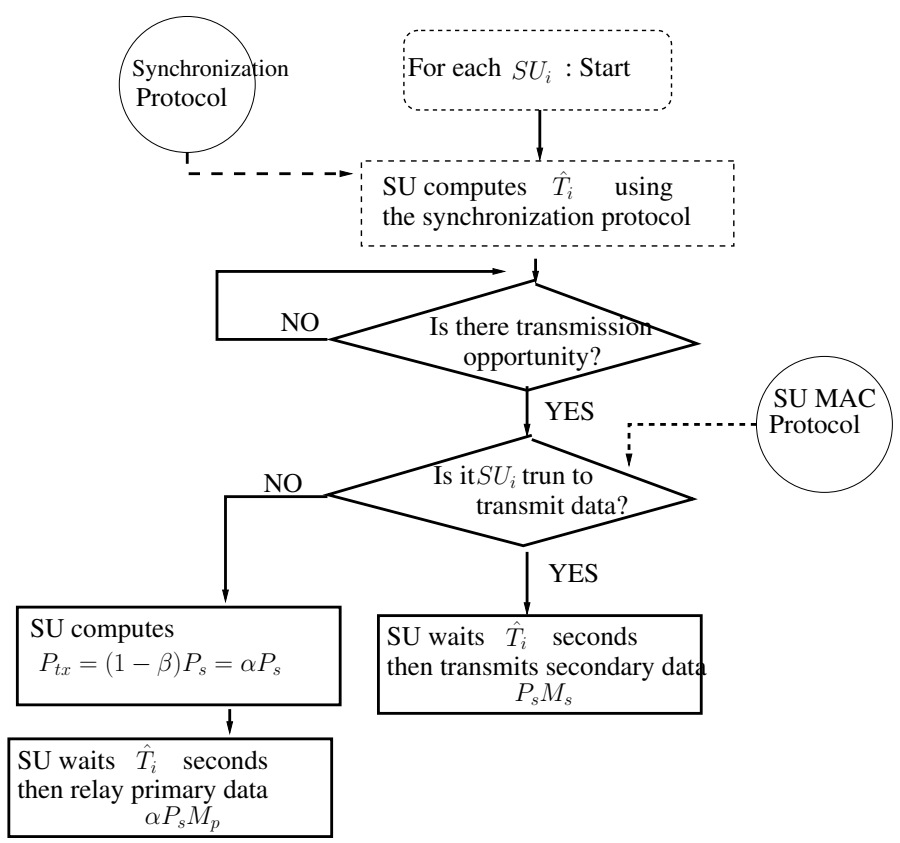

Fig. 8 Flow graph for the Secondary Under Cover protocol. 


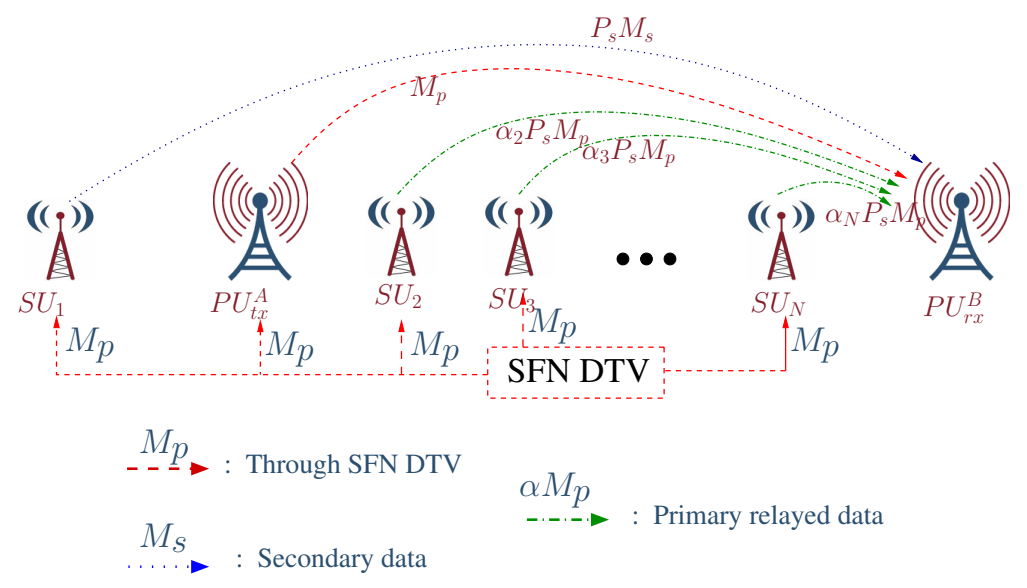

Fig. 4 Extended network scenario with two PUs and multiple SUs is presented, using SFN DTV as the source of primary data for secondary system.

As SUs are located randomly, some will be close to and others will be far from the primary receiver, whose exact location is not known. However, after computing $\hat{T}$, SUs can use this value to estimate their distance from the primary receiver. This will let the SUs in support control and thus minimize power while relaying the primary data: if $\dot{T}$ is large, there is no need for full power to transmit as the primary receiver is expected to be close by. If $\tilde{T}$ is small then higher power is needed, the primary receiver being estimated to be far away. A simple relation to relate $\widehat{T}$ and the required transmission power is as follows:

$P_{t x}=(1-\beta) \times P_{z}=\alpha P_{z}$

Where $\beta_{\epsilon}[0,1]$ is the normalized value of $\hat{T}$ and $\alpha=(1-\beta)$.

Considering secondary data transmission and how the secondary receiver will be able to extract its own data from the primary data, we assume the usage of the Dirty Paper Coding (DPC) technique by both SU transmitter and decoding by SU receiver [13, 14]. DPC is a technique for efficient transmission of digital data through a channel subjected to some interference known to the transmitter. The technique consists of pre-coding the data in order to cancel the effect caused by the known interference, moreover, in information-theoretic terms; dirty-paper coding achieves the channel capacity, without a power penalty and without requiring the receiver to gain knowledge of the interference state. Next, we evaluate the performance of the SUC protocol.

\section{Performance Evaluation}

Given the system model described in section 2 with a network as in Fig. 2, we adopt the transmission scenario of Fig 9, which is an extension of the scenario in Fig. 7. Considering a PU failure probability of $10 \%$, one can see how $5 U_{1}$ uses the opportunity of retransmission to transmit with full power secondary data while other SUs, except for the SU receiver, relay the primary data to cover during the $5 U_{1}$ transmission. 


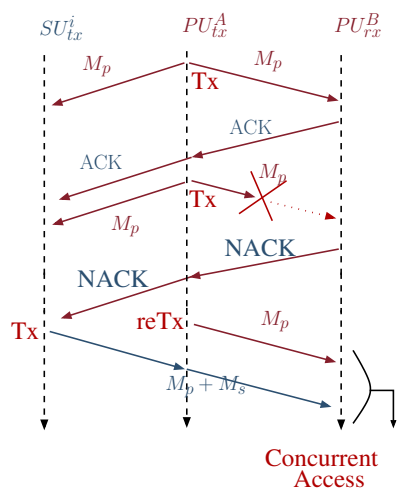

Fig. 3 Message transmission using HARQ protocol is presented between a primary pair (,),

While a is listening and trying to access the primary spectrum concurrently with the primary retransmission, using an overlay access scheme. (,) are the PU's and SU's messages, respectively.

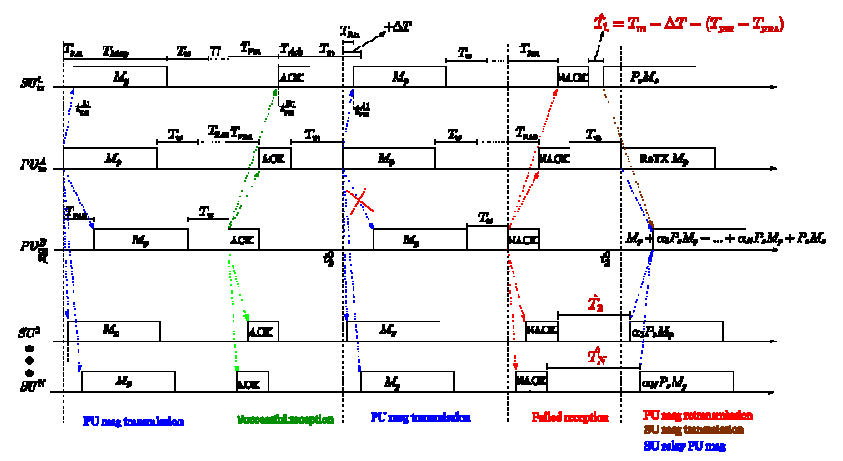

Fig. 9 Transmission scenario using HARQ as the source of primary data for secondary system, using the SUC protocol for interference compensation.

Simulating the extended example scenarios in Fig. 2 with a $10 \%$ failure probability, we examine two cases. In the FAR case, $S U_{t x}$ is farther from the primary receiver than from the primary transmitter. On the other hand, in the CLOSE case, $5 U_{t w}$ is closer to the primary receiver than to the primary transmitter.

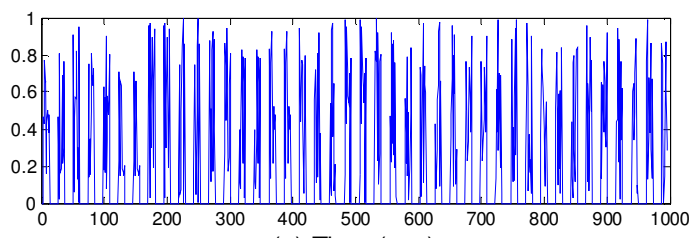

(a) Time (sec)

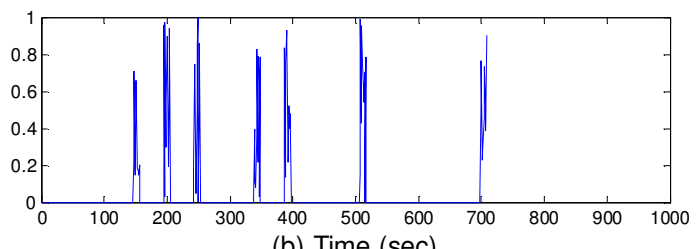

Fig.10 Simulation results for FAR transmission case (a) Reception time from PU's transmission and (b) Reception time from SU transmission. 
Fig. 9, presents a step by step simulation of the FAR case. Looking at the results in Fig. 10 and Fig. 11, one can see how a SU can have the correct estimate of time $\hat{T}$ required to achieve synchronous reception at the PU receiver. With synchronization at the symbol level in time combined with our practical assumptions as in section two, the relayed primary messages will sum coherently with the original primary message from the primary transmitter at the primary receiver. Thus an increase in SINR will be achieved at the primary receiver.

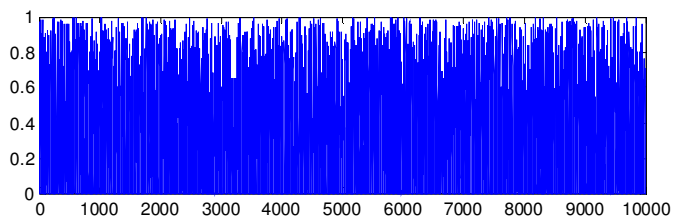

(a) Time (sec)

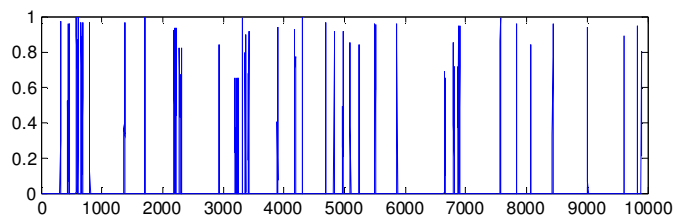

(b) Time (sec)

Fig. 11 Simulation results for CLOSE transmission case. (a) Reception time from PU's transmission and (b) Reception time from SU transmission.

\section{Conclusions}

In this paper we proposed a cooperative dynamic access overlay protocol for cognitive radio networks. Our Secondary Under Cover (SUC) protocol overcomes some drawbacks of the conventional overlay access scheme and makes it an advantage to have a secondary system coexisting within the primary system, as this improves the primary SINR while simultaneously enabling secondary data transmission. In SUC, secondary users do not need to split their power while transmitting and the task of interference compensation is devolved to cooperating secondary users.

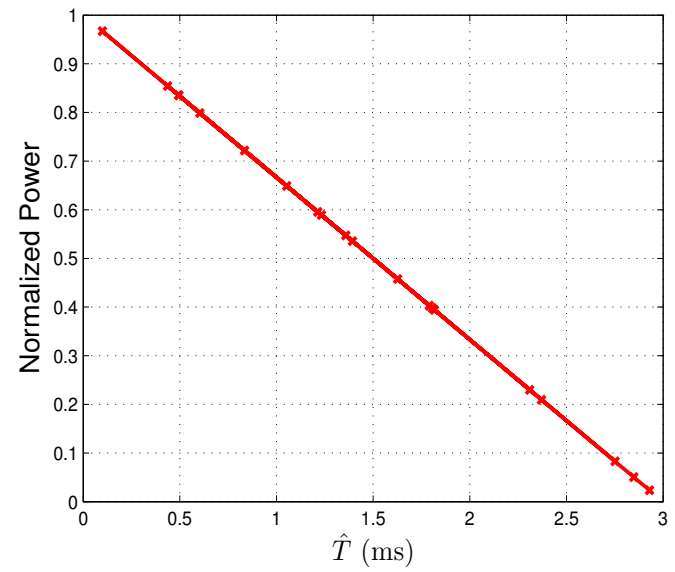

Fig. 12 The relation between the secondary transmission power and the distance from the primary receiver estimated using. 


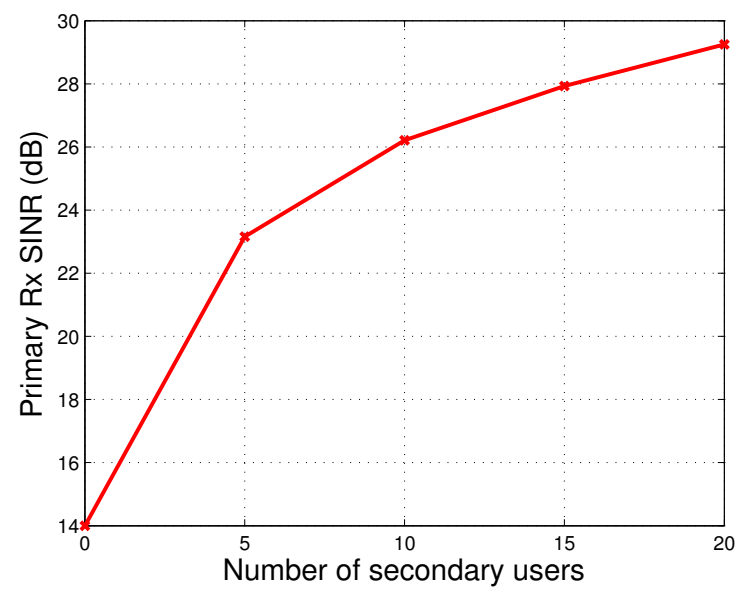

Fig. 13 The relation between the primary SINR and number of SUs in the network

\section{REFERENCES}

[1] Shin, K.G., Ann Arbor, Hyoil Kim, Min, A.W. and Kumar, A, "Cognitive radios for dynamic spectrum access: from concept to reality", Wireless Communications, IEEE, 17: 64 - 74, Dec 2010.

[2] A Masri, S. Tarapiah, and Y. Dama. "Secondary user power saving in overlay cognitive radio networks", International Journal of Computer Applications, 85:13-20, Jan 2014.

[3] S. Srinivasa and S. A. Jafar, "Cognitive radios for dynamic spectrum access - the throughput potential of cognitive radio: A theoretical perspective", Communications Magazine, IEEE, 45(5):73 - 79, 2007.

[4] J. Sachs, I. Maric, and A. Goldsmith. "Cognitive cellular systems within the tv spectrum", In New Frontiers in Dynamic Spectrum, 2010 IEEE Symposium, pages 1 - 12, Singapore, May 2010.

[5] A. Masri, Y. A. S. Dama, A. Musa and F. Hasan, "Distributed Synchronization Protocol for Secondary Overlay Access in Cognitive Radio Networks", Sixth International Conference on Internet Technologies \& Applications (IEEE ITA15 - EERT)", at Wrexham, North Wales, UK, pp. 445-447, 2015.

[6] A. Jovicic and P. Viswanath, "Cognitive radio: an information theoretic perspective", IEEE Trans. Inf. Theory, page 3945 3958, 2009.

[7] A. Goldsmith, S. Jafar, I. Maric, and S. Srinivasa, "Breaking spectrum gridlock with cognitive radios: an information theoretic perspective", pages $894-914,2009$.

[8] A. Rico-Alvarino, C. Mosquera, and F. Gonzalez, "On the coexistence of primary, secondary transmitters in a broadcast network”, In CogART 2011 Barcelona, Catalonia, Spain, 2011.

[9] R. A. Tannious and A. Nosratinia, "Cognitive radio protocols based on exploiting HARQ retransmissions", IEEE Trans. Wirel. Commun., 9(9):2833 - 2841, 2010.

[10] R. D. Souza, "Reducing co-existence penalty of retransmission-based cognitive radio protocol", Electronic letters, 47(6):409 - 411, March 2011.

[11] H. R. Karimi, "Geolocation databases for white space devices in the uhf tv b,s: Specification of maximum permitted emission levels", In New Frontiers in Dynamic Spectrum Access Networks (DySPAN), pages 443 - 454, 2011.

[12] M. Batariere, K. Baum, and T. Krauss, "Cyclic prefix length analysis for 4G OFDM systems", in IEEE Vehicular Technology Conference, VTC2004-Fall, 2004.

[13] M. Costa, "Writing on dirty paper", in IEEE Trans. Information Theory 29 (3): 439-441, May 1983.

[14] C. T. K. Ng and A. Goldsmith, "Transmitter Cooperation in Ad-Hoc Wireless Networks: Does DirtyPaper Coding Beat Relaying?", in IEEE Information Theory Workshop. San Antonio, Texas. pp. 277-282, October 2004. 